Supporting Information

\title{
Light-Responsive Mesoporous Silica Nanoparticles Loaded with Osmanthus Fragrance for
}

\section{Improving Leather Odor}

Xingyu $\mathrm{Hu}^{\dagger}$, Ming $\mathrm{Liu}^{\dagger}$, Yajun Cheng ${ }^{\dagger}$ and Jing $\mathrm{Hu}^{\dagger}{ }^{*}$ *

†School of Perfume and Aroma Technology, Shanghai Institute of Technology, Shanghai, 201418, P.

R. China

\section{Corresponding Author}

*E-mail: hujing@sit.edu.cn, hujing616@126.com.

Table S1

Sensory evaluation of aromatic leather

\section{Evaluation criteria}

\begin{tabular}{|c|c|c|c|}
\hline Pungent odor intensity & Aroma concentration & Aroma comfort value & Evaluation score \\
\hline Strong & Very high & $\begin{array}{c}\text { Comfortable osmanthus scent, with no } \\
\text { leather smell }\end{array}$ & 10 \\
\hline High & High & $\begin{array}{l}\text { Comfortable osmanthus scent, with a } \\
\text { slight leather smell }\end{array}$ & 8 \\
\hline Slightly high & Slightly high & $\begin{array}{c}\text { The overall aroma is is average and } \\
\text { comfortable, with a leather odor }\end{array}$ & 6 \\
\hline Low & Low & $\begin{array}{l}\text { The oveal fragrance is less comfortable, } \\
\text { and the leather smell is high }\end{array}$ & 4 \\
\hline hardly perceivable & hardly perceivable & $\begin{array}{l}\text { The overall scent is uncomfortable and } \\
\text { cannot cover up the smell of leather }\end{array}$ & 2 \\
\hline Not perceivable & Not perceivable & The aroma is not perceivable & 0 \\
\hline
\end{tabular}


Table S2

GC-MS results of three leather samples

\begin{tabular}{|c|c|c|c|c|}
\hline \multirow{2}{*}{ Odor classification } & \multirow{2}{*}{ Compounds } & \multicolumn{3}{|c|}{ Content $\left(\mathrm{mg} \mathrm{m}^{-3}\right)$} \\
\hline & & Leather & Resin leather & Aromatic leather \\
\hline \multirow{3}{*}{ Amine odor } & $\begin{array}{c}\mathrm{N}-h e x y l \\
\text { methylamine }\end{array}$ & 8.45 & 6.83 & 0.4 \\
\hline & $\begin{array}{c}\mathrm{N} \text {-methyl } \\
\text { isopropylamine }\end{array}$ & 14.9 & 12.52 & 6.9 \\
\hline & Diethylenetriamine & 3.93 & 3.16 & 3.26 \\
\hline \multirow{6}{*}{ Chemical reagent } & Toluene & 0.26 & 4.95 & 1.27 \\
\hline & P-xylene & 0.06 & 0.06 & 0.42 \\
\hline & $\begin{array}{c}\text { Hexamethylcyclotet } \\
\text { rasiloxane }\end{array}$ & 0.85 & 0.67 & 1.81 \\
\hline & $\begin{array}{c}\text { Octamethylcyclotris } \\
\text { iloxane }\end{array}$ & 0.76 & 0.71 & 0.3 \\
\hline & Dodecane & 1.59 & 1.47 & 0.7 \\
\hline & Benzaldehyde & 0.13 & 0.35 & 0.53 \\
\hline \multirow{10}{*}{ Pungent } & Styrene & - & 0.22 & 0.53 \\
\hline & O-cymene & 0.88 & 1.89 & 2.18 \\
\hline & Dichloromethane & 1.28 & 0.29 & 0.74 \\
\hline & Trichloromethane & 0.34 & 0.18 & 1.21 \\
\hline & 2-octanone & 0.48 & 0.48 & 0.5 \\
\hline & $\begin{array}{c}\text { 2,6-Di-tert-butyl p- } \\
\text { cresol } \\
\text { 1,2,3,4-tetrahydro- }\end{array}$ & - & 0.2 & 0.28 \\
\hline & $\begin{array}{c}1,1,6- \\
\text { trimethylnaphthalen } \\
\mathrm{e}\end{array}$ & 0.59 & 0.67 & 0.09 \\
\hline & $\begin{array}{c}\text { 1,1,6-Trimethyl-1,2- } \\
\text { dihydronaphthalene } \\
\text { 3,3-Dimethyl-4- }\end{array}$ & 0.14 & 0.13 & 0.22 \\
\hline & $\begin{array}{l}\text { methylamino-2- } \\
\text { butanone }\end{array}$ & 4.52 & 1.52 & 1.62 \\
\hline & Benzothiazole & 0.04 & 0.22 & 0.29 \\
\hline \multirow[b]{2}{*}{ Woody } & $\alpha$-celinene & 0.62 & 0.42 & 0.26 \\
\hline & $\begin{array}{c}2,4- \\
\text { Dimethylstyrene }\end{array}$ & 0.14 & 0.96 & 1.71 \\
\hline \multirow{3}{*}{ Fruity } & Butyl Butyrate & - & 0.84 & 1.86 \\
\hline & D-limonene & 13.28 & 25.75 & 18.26 \\
\hline & Butyl caproate & - & 1.59 & 0.47 \\
\hline \multirow{2}{*}{ Green } & Hexanal & 0.46 & 2 & 1.05 \\
\hline & Phytol benzoate & - & - & 0.32 \\
\hline \multirow{10}{*}{ Floral } & 2-ethylhexanol & - & 0.33 & 0.27 \\
\hline & Linalyl Acetate & - & - & 0.48 \\
\hline & Linalool & - & - & 5.48 \\
\hline & trimethylcyclohexyl) & - & - & 6.32 \\
\hline & -2-butanone & & & \\
\hline & Dihydro-a-ionone & - & - & 1.06 \\
\hline & Dihydro- $\beta$-ionone & - & - & 7.22 \\
\hline & a-ionone & - & - & 3.41 \\
\hline & $\beta$-ionone & - & - & 5.95 \\
\hline & 3-butene-2-one & - & - & 0.34 \\
\hline
\end{tabular}




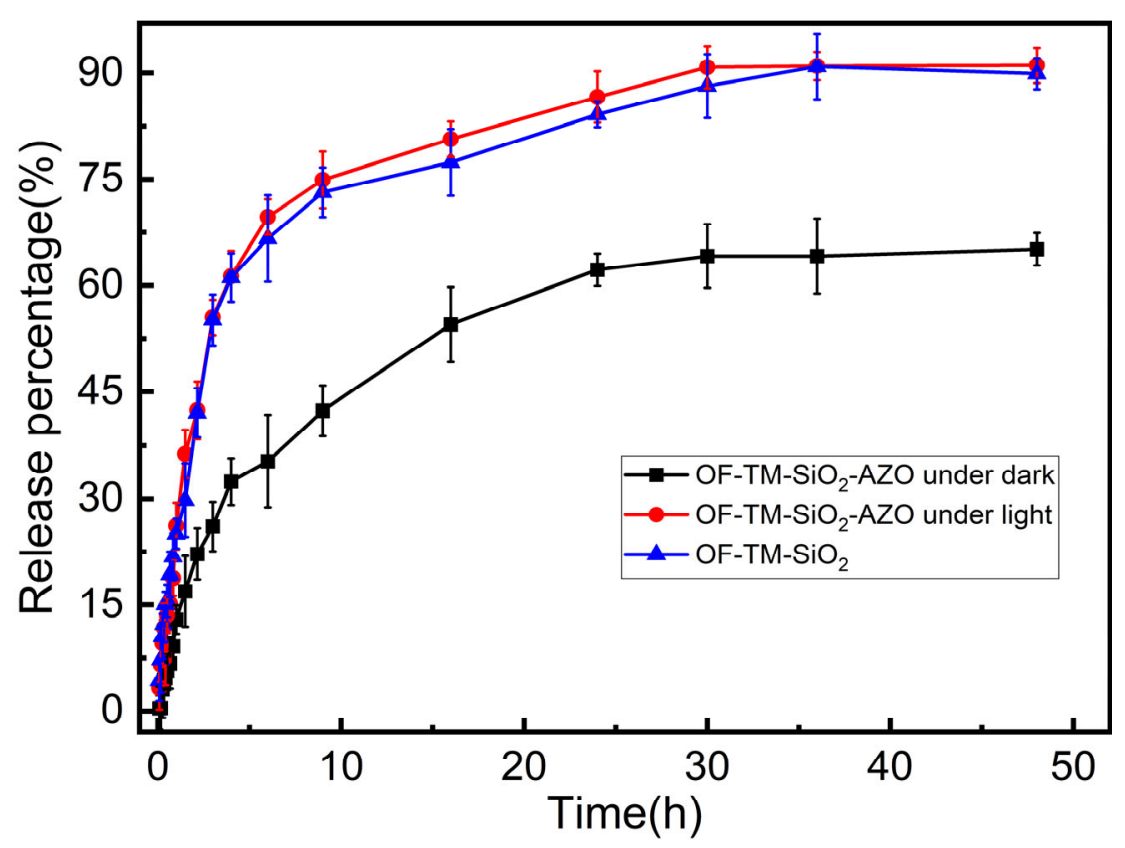

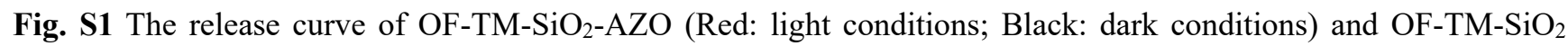
without azobenzene residues (blue). Data are shown as mean \pm s.d., with $n=3$. 